\title{
Mehr als nur ein neuer Name
}

\section{Julia Dratva}

Präsidentin Schweizerische Gesellschaft für Prävention und Public Health, ZHAW Departement Gesundheit, Institut für Gesundheitswissenschaften

\author{
Die Schweizerische Gesellschaft der Fachärztinnen und Fachärzte für Prävention \\ und Gesundheitswesen heisst ab jetzt in allen Landesregionen gleich - Gesund- \\ heitswesen wird mit Public Health ersetzt. Hinter dem Namenwechsel steht mehr \\ als ein paar Buchstaben. Seit 2015 hat sich die Gesellschaft neu aufgestellt - new \\ public health möchte man meinen!
}

Die Schweizerische Fachgesellschaft für Prävention und Public Health, ehemals Schweizerische Fachgesellschaft für Prävention und Gesundheitswesen, ist die Dachorganisation der Fachärztinnen und Fachärzte für Prävention und Public Health sowie zahlreicher weiterer Ärztinnen und Ärzte, die im Bereich Public Health tätig sind und einem gemeinsamen Leitbild verpflichtet sind. Sie wurde 1976 als Verein gegründet. In den vergangenen Jahren hat sich die Fachgesellschaft unter der Präsidentschaft von Dr. med. Karin Faisst und ab 2016 von Prof. Dr. med. Julia Dratva neu aufgestellt und wesentliche Weiterentwicklungen und Aktivitäten umgesetzt. Die Vereinheitlichung des Namens in allen Landesteilen mit sanità pubblica, santé publique und Public Health ist da nur das Tüpfelchen auf dem i. Sie steht für das Verständnis unserer Fachorganisation, sich gesellschaftlich, politisch und standespolitisch für die Gesundheit der gesamten Bevölkerung in der Schweiz einzusetzen.

\section{In den vergangenen Jahren hat sich die Fach- gesellschaft neu aufgestellt.}

Die demographische Entwicklung und die Zunahme chronischer Erkrankungen, der Fachkräftemangel und die Kostensteigerung stellen das Gesundheitssystem vor grosse Herausforderungen. Dieser zunehmenden Belastung sowohl des Gesundheitssystems als auch der Bevölkerung begegnet die Schweiz unter anderem mit Gesundheitsstrategien, z.B. NCD-Strategie, Aktionsprogramm zur Suizidprävention, Gesundheitsstrategie 2020, sowie mit neuen Versorgungsmodellen (z.B. integrierte Versorgung). Die Strategien verfolgen dabei ein ganzheitliches Verständnis von Gesundheit unter Berücksichtigung der physischen, psychischen, sozialen und umweltbedingten Einflussfaktoren in verschiedenen Lebensphasen. In Anlehnung an die "triple aims» von Berwick et al. (2008) stehen drei übergeordnete Ziele im Vordergrund: 1. die Verbesserung der Patientenzufriedenheit und der Versorgungsqualität (improving the experience of care, including quality and satisfaction), 2. die Verbesserung der Bevölkerungsgesundheit (improving the health of populations) sowie 3. die Reduktion der Gesundheitskosten (reducing per capita costs of health care) (Berwick 2008).

Als Schweizerische Fachgesellschaft für Prävention und Public Health sehen wir unsere zentrale Aufgabe in der Weiterbildung von ärztlichen Fachpersonen, um diesen Herausforderungen zu begegnen und zu Lösungen beizutragen. Daher setzt sich die Fachgesellschaft für eine bedarfsorientierte und innovative Weiterbildung ein. Während der Blick in andere europäische Länder schnell deutlich macht, wie viel wirkungsvoller ärztliche Public-Health-Kompetenzen eingesetzt werden könnten, müssen wir dafür in der Schweiz noch an der Erkenntnis und der Umsetzung arbeiten. Innovation in der Weiterbildung bedeutet, nicht nur neue Ansätze und Wege zu probieren, sondern auch und vor allem Ärztinnen und Ärzte für die künftigen grossen Herausforderungen auszubilden. In diesem Sinne haben die Experten im Rahmen der kürzlich erfolgreich abgeschlossenen Akkreditierung unseres Faches die Schweizerische Fachgesellschaft für Prävention und Public Healthaufgefordert, "global public health» in der Weiterbildung noch stärker zu beachten als bislang. Etwa 40 Prozent der Titelträger und Titelträgerinnen arbeiten an Hochschulen (Universitäten und Fachhochschulen), 40 Prozent sind in öffentlichen Institutionen wie kantons- oder schulärztlichen Diensten oder Registern tätig, und weitere 20 Prozent sind bei Gesundheitsligen, Versicherern oder weiteren PublicHealth-Institutionen engagiert. Mit Blick auf diese Vielfalt der Berufsfelder begrüsst die Fachgesellschaft eine Zunahme nichtuniversitärer Weiterbildungsstät- 
ten, um verstärkt die verschiedenen Arbeitswelten von Fachärztinnen und Fachärzten widerzuspiegeln.

Die fachspezifische Weiterbildung zur Fachärztin und zum Facharzt für Prävention und Public Health zeichnet sich durch einige Besonderheiten aus, mit denen

\section{Grundstein der Weiterbildung sind mindestens zwei Jahre klinische Tätigkeit.}

diese sich von anderen Experten im Gesundheitswesen abheben. Grundstein der Weiterbildung sind mindestens zwei Jahre klinische Tätigkeit. Ärztliche Verantwortung für Patientinnen und Patienten sowie das Gesundheitswesen von innen zu kennen ist eine wesentliche Stärke der Public-Health-Ärzte und -Ärztinnen gegenüber anderen Public-Health-Fachpersonen. Die fachspezifische theoretische Weiterbildung besteht in der Regel aus dem Erwerb des Masters of Public Health (MPH) oder eines Masters in Epidemiology (MSc Epidemiology). Diese fachspezifische theoretische Weiterbildung dauert ein Jahr und vertieft die Fachkenntnisse der Kandidierenden in drei Themengebieten:

Epidemiologie und Biostatistik (Datenanalyse und Praxistransfer), Prävention und Gesundheitsförderung (Problemanalyse bis hin zur Evaluation von Interventionen) sowie Gesundheitsversorgung (Gesundheitspolitik, Gesundheitsökonomie, Gesundheitsrecht).

Das neue Weiterbildungsprogramm der SPPH (Revision Juni 2017) orientiert sich an den empfohlenen $\mathrm{Pu}$ -

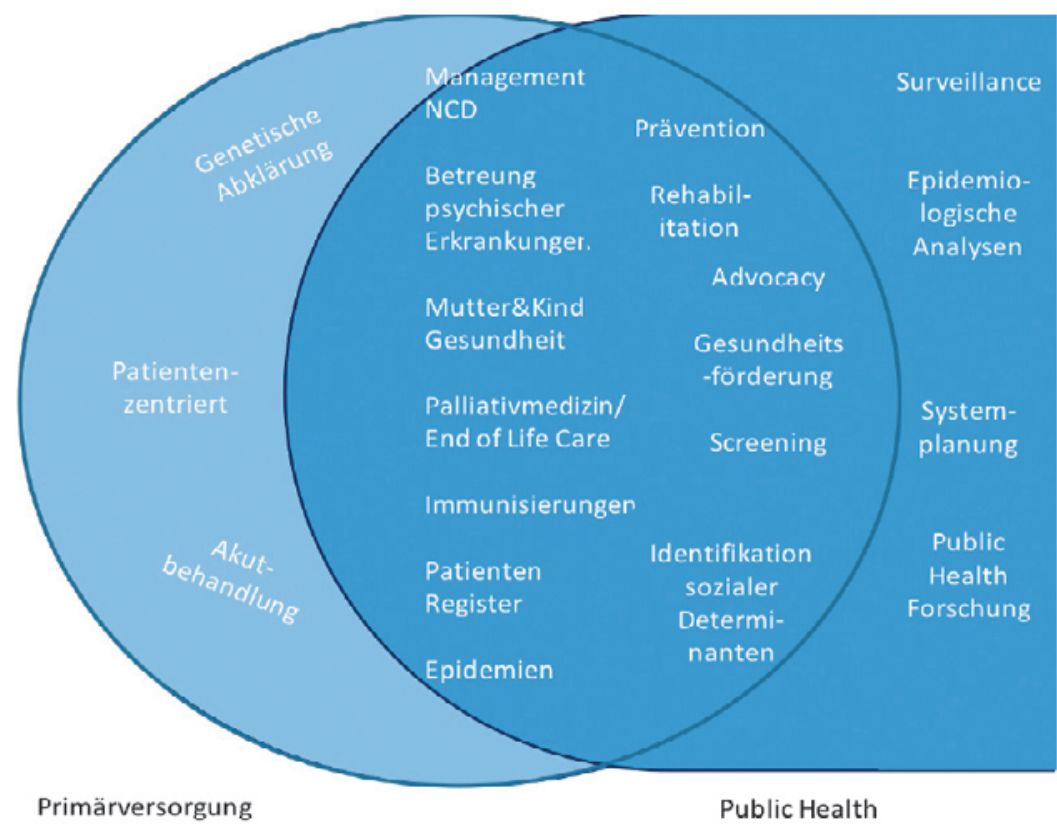

Individuum
blic-Health-Kompetenzen der Association of Schools of Public Health in the European Region (ASPHER, https:// www.aspher.org/). Diese international anerkannten Lerninhalte sind auch eine gute Leitlinie zur Berücksichtigung verschiedener Ausbildungsverläufe. Die zunehmende Heterogenität der theoretischen Ausbildungen ist eine Herausforderung, der wir uns gerne stellen. Wir betrachten diese Diversität als Bereicherung und wünschen uns, den Kreis unserer Mitglieder um kompetente ärztliche Public-Health-Spezialistinnen und -Spezialisten zu erweitern. Seit kurzem wird z.B. der Master für Versicherungsmedizin als theoretische Weiterbildung unter Auflagen anerkannt, und Versicherungsmediziner und -medizinerinnen können, vorausgesetzt sie erfüllen die anderen Weiterbildungskriterien, die Facharztprüfung in Prävention und Public Health ablegen.

\section{Die zunehmende Heterogenität der theoreti-} schen Ausbildungen ist eine Herausforderung, der wir uns gerne stellen.

Public Health hat ebenso wie die Primärversorgung eine tragende Rolle in der Gesundheitsversorgung der Bevölkerung. Achesons Definition von Public Health macht deutlich, wie viel beide Bereiche gemein haben: Public Health ist "the science and art of preventing disease, prolonging life and promoting health through the organized efforts of society" (Breda et al. 2015). PublicHealth-Fachärztinnen und -ärzte bewegen sich auf verschiedenen Ebenen der Gesundheitsversorgung. Einerseits sind sie auf der Makroebene eingebunden, bei der Planung und Entwicklung eines zukunftsfähigen, nationalen Gesundheitssystems, und andererseits auf der Mesoebene, bei der Umsetzung auf Gemeindeund kantonaler Ebene. Auf der Mikroebene begegnen Public-Health-Fachleute Individuen und Bevölkerungsgruppen mit speziellen Bedürfnissen oder Risiken, zum Beispiel in Gesundheitsförderungsprojekten oder im Rahmen von schulärztlichen Abklärungen. Die Primärversorgung ist vornehmlich auf der Mikroebene aktiv im direkten Kontakt mit den individuellen Patientinnen und Patienten und ihrem Umfeld: Diagnose, Therapie und Prävention. Wenngleich eine Vielzahl der Aufgabenbereiche von Public Health und Primärversorgung gleich sind, sind die Rollen und Funktionen darin andere (Abb. 1). Zunehmend wird die Primärversorgung zu übergeordneten Aufgaben direkter beitragen, zum Beispiel im Gesundheitsmonitoring oder in der Gesundheitsförderung (siehe NCD-Strategie). Damit wären zukünftig weitere Aufgabenbereiche überlappend. 
Vernetzung und Kommunikation sind daher zentral. In diesem Sinne pflegen wir enge Kontakte zum Beispiel mit dem Departement für Public Health der FMH, der Vereinigung der Kantonsärzte oder dem Verein der Schweizerischen Schulärzte, um ein paar der ärztlichen Partnerorganisationen zu nennen, mit denen wir mittels gemeinsamer Fortbildungen und Gesprä-

Der regelmässige Kontakt unter Ärztinnen und Ärzten anderer Disziplinen dürfte noch gestärkt werden, sowohl inhaltlich als auch politisch.

che im Austausch stehen. Der regelmässige Kontakt unter Ärztinnen und Ärzten anderer Disziplinen dürfte noch gestärkt werden, sowohl inhaltlich als auch politisch. Auch deswegen bieten wir Ärztinnen und Ärzten mit einem anderen Facharzttitel, die sich als PublicHealth-Ärztinnen und -Ärzte verstehen oder Public Health generell unterstützen wollen, eine ausserordentliche Mitgliedschaft an (http://sgpg.ch/sgpg_d/ mitglied-werden/). Die verschiedenen Perspektiven auf Gesundheit und Krankheit zusammenzubringen ermöglicht, besser an einem Strick zu ziehen, wenn es um die Sicherstellung eines hochwertigen und fairen Gesundheitssystems und den Schutz vor Krankheitsrisiken geht.

Public Health ist nicht nur interdisziplinär unterwegs, sondern auch in höchstem Masse ein interprofessionelles Fach. Und das war es schon vor dem Hype der letzten Jahre um das Thema Interprofessionalität. In der Schweiz ist Public Health Schweiz (www.publichealth.ch), der nationale interprofessionelle Fachverband, nach der FMH unser Hauptpartner. Ebenfalls von grosser Wichtigkeit ist eine enge Zusammenarbeit mit der Swiss School of Public Health (https://ssphplus. ch/), einer interuniversitären Fakultät aller acht Schweizer Universitäten zur Förderung der postgradualen Ausbildung und Wissenschaft in Public Health. Im Ausland sind wir mit der Österreichischen Gesellschaft für Public Health, der Vorarlberger Public Health Gesellschaft, der Deutschen Gesellschaft für Sozialmedizin und Prävention sowie der Deutschen Public Health Gesellschaft vernetzt. Dank der zweijährlichen Tagung «Public Health ${ }^{3}$ » $\mathrm{PH}^{3}$, http://sgpg.ch/?s=PH3 ) können wir dringende Fragen der öffentlichen Gesundheit grenzüberschreitend diskutieren. Die nächste $\mathrm{PH}^{3}$-Tagung findet übrigens im Januar 2020 erneut im Bodenseeraum statt - alle Public-Health-Interessierten seien dazu schon mal herzlich eingeladen! Wer als starke Stimme in der Gesellschaft gehört werden möchte, muss hin und wieder laut werden. Als kleine Fachgesellschaft gelingt auch das am besten im Chor, sprich in Kooperation. Gemeinsam mit allen Fachgesellschaften setzen wir uns für ein zukunftsfähiges Gesundheitssystem und für die Gesundheit aller Bevölkerungsgruppen ein. Die Herausforderungen sowohl medizinischer, sozialer, umweltbezogener als auch gesundheitsökonomischer Art sind nur so zu lösen. Die Kombination von individualmedizinischen und populationsmedizinischen Kompetenzen und Argumenten wird kurz- und langfristig die grösste Wirkung haben.

\section{Literatur}

- American Academy of Family Physicians (AAFP). Integration of Primary Care and Public Health (Position Paper). 2017.

- Breda J, Wijnhoven T, Gabrijelčič M, Sigfrid L. Facets in Public Health in Europe. 2015.

- Berwick DM, Nolan TW, Whittington J. The triple aim: care, health, and cost. Health Aff (Millwood). 2008;27:759-69. 